\title{
Hybrid Computations Using Continuum and Molecular Dynamics for Micro-fluidics
}

\author{
Jia Cui ${ }^{1}$, and Guowei $\mathrm{He}^{1,2, *}$ \\ ${ }^{1}$ LNM, Institute of Mechanics, CAS, China \\ ${ }^{2}$ Department of Aerospace Engineering, Iowa State University, U.S.A.
}

\begin{abstract}
A hybrid numerical method of particle-continuum dynamics designed for micro-fluidics is being developed which solves the Navier-Stokes equations in one domain, where the continuum description is still valid, and uses the molecular dynamics in another domain where the continuum description fails. The matching of these two descriptions can be achieved by a constrained particle dynamics in the overlap region, which exchanges the information between the molecular dynamics and the Navier- Stokes equations. However, the existing models for the constraint particle dynamics often have the empirical parameters such as the coupling parameter, the size of overlap domain and the ratio of the time steps for the molecular dynamics and the Navier-Stokes equation. In this paper, we develop a dynamic Langevin equation, and the coupling parameters in the models can be determined by the velocity consistent constraint. The dynamic scheme is used to simulate the sudden-start Couette flows with either slip or no-slip boundary conditions. The results obtained are quantitatively in agreement with the analytical solutions under the no-slip boundary condition and the full MD simulations under the certain slip boundary conditions. This agreement demonstrates the validity of the dynamic Langevin equation.
\end{abstract}

Keywords-hybrid; molecular dynamics; continuum; Langevin

\section{Introduction (HEADING l)}

The fluid flows in micro fluidics involve a wide range of scales from the atomistic scales to the continuum ones. Usually, the Navier-Stokes (NS) equations are used to describe the fluid flows at the continuum scales, and the molecular dynamics are used to describe the flows in the small regions near interfaces. In the continuum description, no-slip boundary conditions are often employed at the solid-fluid interfaces, which have been proven to be effective and successful in most of the fluid flows at macro scales. However, in micro fluidics, the no-slip boundary conditions may not be valid at the solid-fluid interfaces $[1,2]$. Some small structures in the solid-fluid interfaces that can be ignored at macro scales may cause large changes for the flows in the micro fluidics [3].

Particle methods, such as molecular dynamics (MD), are able to solve the problems mentioned above. However, it's not realistic to use the full $\mathrm{MD}$ simulations for experimental or industrial designs because of the limitations of current computer's capability. To solve the problem, a hybrid method of particle-continuum dynamics is being developing [3-6]. In the hybrid method, the MD simulations are used in the region where the continuum description fails, for example, in the small region around the interfaces where the no-slip boundary conditions may not hold true [1-3]. Meanwhile, in the

This work was supported by Chinese Academy of Sciences under the innovative project "multi-scale modeling and simulation in complex system" and National Natural Science Foundation of China (Project code 10325211).

*Contact author: hgw@lnm.imech.ac.cn. remaining regions, the fluid flow can be described by the NS equations, see Fig. 1 for the sketch. The hybrid method could save much more computational time than the full MD simulations in the entire region. To match the results from the MD and the NS equations, a coupling scheme has to be introduced. The coupling scheme could be achieved via a constrained particle dynamics in the overlap region, where the MD simulations exchange information with the NS equations. The constrained particle dynamics are constructed in order to guarantee the continuity of the physical quantities of interests, such as mass, momentum, energy and their fluxes.

Several coupling schemes have been developed for the hybrid method. O'Connel and Thompson [7] suggest a Langevin particle dynamics with an empirical parameter. The empirical parameter determines the coupling intensity between the MD and the NS equations. Our experience with the Langevin dynamics indicates that the results obtained from the hybrid method are very much determined by the parameter. Flekkoy et al. [8-13] propose to couple the momentum through fluxes for the steady and unsteady flows. Recently, Nie et al. $[14,15]$ developed a coupling model of the consistent velocity fields between the MD and NS equations, using a constrained particle dynamics. Among all of the coupling schemes, there exist some unavoidable free parameters, such as the size of the overlap region, the ratio of time steps between the $\mathrm{MD}$ and the NS equations and so on. These free parameters have to be given empirically for the outputs. So far, there has been no any general rule to determine them a priori.

In this paper, we will develop a dynamic Langevin equation to couple the MD and the NS equations. In the novel approach, the particles in the overlap region follow the Langevin equations, which is similar to the O'Connel and Thompson's

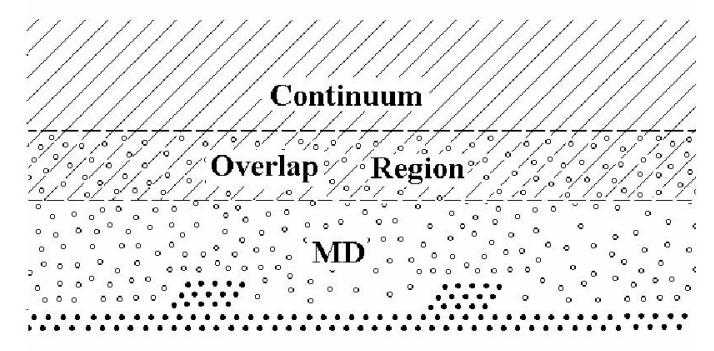

Figure 1. A schematic diagram for the hybrid method. Circles indicate particle region: empty ones represent the fluid and solid ones represent the solid substrate with some nano- or micro- structure. Diagonals indicate the continuum region. The overlap region is between the two dashed lines. 
model. The free parameter in the Langevin equation is dynamically adjusted in terms of the velocity constraint. Therefore, the coupling intensities may be different at different locations and different time steps. The new scheme will be verified against the sudden-started Couette flows, and the results obtained will be compared with the analytical solutions under the no-slip boundary condition and the full MD results under the certain slip boundary conditions. The comparisons show the accuracy and the potential applications of the dynamic Langevin equation.

\section{HYBRID METHOD}

We start with the introductions of the two methods for describing the fluid flow. In the continuum region, the NS equations for incompressible fluid are written as

$$
\begin{gathered}
\nabla \cdot \boldsymbol{u}=0 \\
\partial \boldsymbol{u} / \partial t+\boldsymbol{u} \cdot \nabla \boldsymbol{u}=-(1 / \rho) \nabla p+\nu \nabla^{2} \boldsymbol{u}
\end{gathered}
$$

Here $\boldsymbol{u}$ is the velocity of the fluid, $\rho$ the density, $\boldsymbol{p}$ the pressure and $v$ the kinematic viscosity. A two-dimensional flow in the $x-z$ plane is considered and the equations are solved numerically by the projection method with the staggered grids that is shown in Fig. 2.

As it is shown in the Fig. 2, we will get the boundary conditions for solving the NS equations by the statistic values from the MD simulations in the hybrid method.

In the discrete region, the MD simulations are implemented. The interaction potential of particles is given by the Lennard-Jones potential

$$
V^{L J}(r)=4 \varepsilon\left[\left(\frac{\sigma}{r}\right)^{12}-\left(\frac{\sigma}{r}\right)^{6}-\left(\frac{\sigma}{r_{c}}\right)^{12}+\left(\frac{\sigma}{r_{c}}\right)^{6}\right],
$$

where $\varepsilon$ and $\sigma$ are the characteristic energy and length scales separately, $r$ is the distance between two particles and $r_{c}$ is the length to cut off the interaction between particles. The equations of motion are integrated using the Verlet scheme. The temperature of the fluid is maintained to be constant. And this is achieved by using a Langevin thermostat in the MD region.

As it is shown in Fig. 1, the overlap region is introduced to achieve the coupling of the two different methods. In the view of the momentum continuity across the MD region and the continuum region, the constraint can be expressed as

$$
<m_{i} v_{i}>=M_{J} u_{J}
$$

where $m_{i}$ is the mass of the $i$ th particle in the Jth cell, $v_{i}$ the particle's velocity, $<>$ the symbol of ensemble average, $u_{J}$ the average continuum velocity from the continuum description and $M_{J}$ the total mass of the fluid in the Jth cell. The

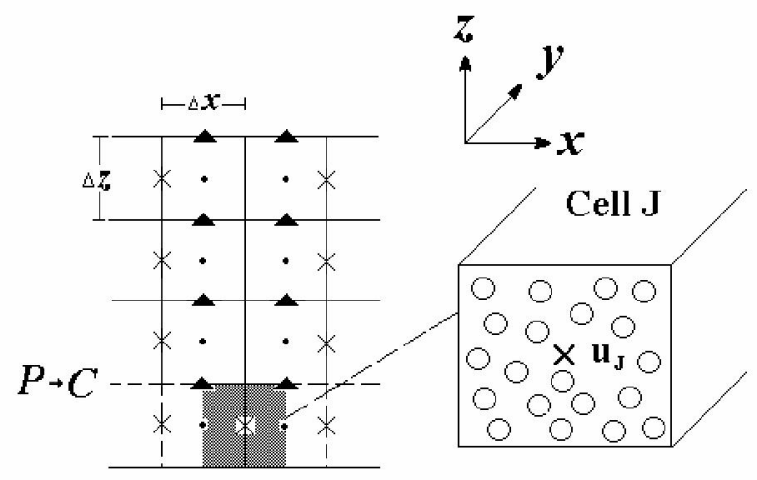

Figure 2. A schematic diagram for the staggered grids and the boundary conditions in the continuum region. Cross and triangle indicate $x$ and $z$ components of the velocity, respectively. $\Delta x$ and $\Delta z$ are the length of the cell in $x$ and $z$ directions, respectively. $P \rightarrow C$ is the lower surface of the continuum region where the boundary conditions for solving the NS equations are needed $u$ is the velocity at the center of the cell $\mathrm{J}$ which will be given by the MD simulations in the hybrid method.

constraint implies that the averaged particle momentum should be equal to the one obtained by continuum method. We achieve this constraint by imposing the O'Connel and Thompson model to the dynamics of the particles in the overlap region. In the conventional MD simulations, equation of motion is

$$
\ddot{x}_{i}=\boldsymbol{F}_{i} / m \text {, }
$$

where $x_{i}$ is the displacement of the particle $i$, and $\boldsymbol{F}_{i}$ is the force brought by the Lennard-Jones potential with the expression

$$
F_{i}=-\sum_{j \neq i} \partial V^{L J} / \partial r_{i}
$$

According to the Langevin equations, a constraint force is brought into the motion equation to achieve the constraining to the dynamics of the particles in the overlap. Thus, the motion equation can be rewritten as

$$
\ddot{x}_{i}=F_{i} / m+\xi \cdot\left(u_{J}-\sum_{j=1}^{N_{J}} \dot{x}_{j} / N_{J}\right),
$$

where $\xi$ is a coefficient which will be dynamically determined by the momentum constraint in the computational process. The dynamic scheme is more reasonable than adopting a fixed parameter in the whole process, since the strength of the force may change in the process from the initial stage to the steady state. When the velocity from the MD simulations is consistent with the one from the continuum method, the constraint force vanishes. We sum up the values of the particles in a cell $\mathrm{J}$ and get the expression of the parameter $\xi$ : 


$$
\xi_{J}(t+1)=\frac{\frac{1}{N_{J}} \sum\left(\ddot{x}_{i}(t)-\frac{F_{i}(t)}{m}\right)}{u_{J}(t)-\frac{1}{N_{J}} \sum_{j=1}^{N_{J}} v_{j}(t)} .
$$

The value is different in each cell and in each computational step according to the momentum constraint from the two regions. To avoid the parameter being too small or two large, we will set a reasonable range for the coupling parameter. To maintain the constant temperature $T$, the Langevin thermostat is used in the overlap region as well.

Another important effect of the particles in the overlap region is to achieve the mass continuity. It is achieved by imposing particles to move across the boundary of the particle description region. Because this only happens in the thin layer near the boundary, we define a constrained layer which is sketched in Fig. 3 to complete the process.

Fig. 3 shows the locations of the overlap region and the thin constrained layer adjacent to the interface $C \rightarrow P$. In this layer, mass flux is imposed, i.e., a certain number of particles are inserted into or removed from the MD region according to the mass flux evaluated by the NS equations. The number of the particles that should be imposed to moving across the boundary is

$$
n=-A \rho u_{z} \Delta t_{F D} / m
$$

where $A$ is the area of the cell boundary that are perpendicular to the interface $C \rightarrow P$ and $\triangle t_{F D}$ is the time step for solving the NS equations. If $n$ is positive, $n$ particles are inserted at the regular intervals over the time unit $\triangle t_{F D}$. They are placed near the interface $C \rightarrow P$ in $z$ direction and randomly in $x$ and $y$ directions. To prevent the distance between the particles inserted and previous from being too small, we will give a position distribution again if it happens. The initial velocities of these inserted particles are equal to the continuum velocity at the $C \rightarrow P$ surface to ensure that the average of micro-velocities is consistent with the macro-velocity. If $n$ is negative, $n$ particles that are closest to the interface $C \rightarrow P$ are removed. At each interval $\triangle t_{F D}$, the nearest integer of $n$ is taken.

Furthermore, to obtain the momentum flux continuity, an external force in $z$ direction should be added on the particles in the constrained layer. The sum of the external forces to the particles in a cell should be determined by the following equation:

$$
\sum_{i} F_{i}^{\prime}=A\left(-P+2 \mu \frac{\partial u_{z}}{\partial z}\right)
$$

Here $\boldsymbol{F}_{\boldsymbol{i}}$ is the external force acting on particle $i, P$ is the local pressure and $u_{z}$ is the velocity in $z$ direction obtained by solving the NS equations. Equation (10) shows that the forces

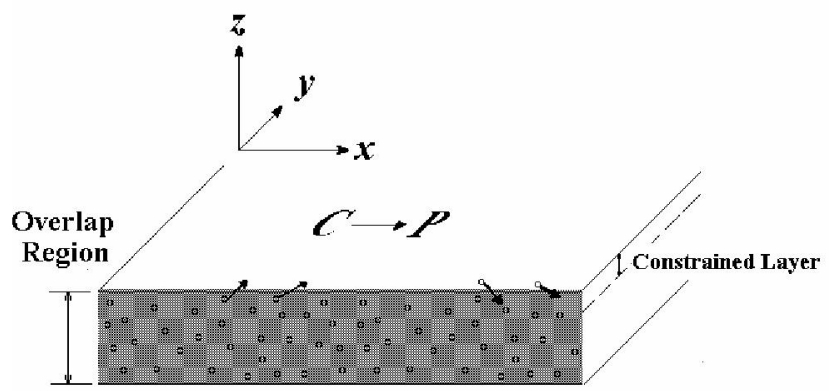

Figure 3. A schematic diagram of the constrained layer. The surface $C \rightarrow P$ is the boundary of the particle description region. Arrows sketch the moving directions of the particles.

on the particles in the constrained layer are determined by the continuum dynamics. To prevent the particles from spreading freely away from the MD region, we introduce an expression of $F_{i}$ as

$$
F_{i}^{\prime}=\frac{g\left(z^{i}\right)}{\sum_{j \in V} g\left(z^{i}\right)} F
$$

Here $F$ denotes the right-hand part of $(10)$ and $g\left(z^{i}\right)$ is an arbitrary weight function which depends on the particle's position in $z$ direction. This function should ensure that the external force for the particles at the interface $C \rightarrow P$ is approaching infinite and for the particles on the bottom surface of the constrained layer is equal to zero. Thus particles are effectively constrained in the MD domain.

\section{RESULTS}

We use the hybrid method to simulate the two dimensional (in the $x-z$ plane) liquid flow. The liquid we choose has the physical parameters as follows: the mass of each atom is $m$, the density is $\rho=0.81 \mathrm{~m}^{-3}$, the cut-off length $r_{c}$ is equal to $2.2 \sigma$, the characteristic time is $\tau=\left(m \sigma^{2} / \varepsilon\right)^{1 / 2}$, the temperature of the fluid is maintained to be $T=1.1 \varepsilon / k_{B}$, where $k_{B}$ is Boltzmann's constant, the damping rate of the Langevin thermostat is $\tau^{-1}$, and at these given $T$ and $\rho$, the fluid is in a well-defined liquid phase with $\mu=2.14 \& \quad \tau \quad \sigma^{-3}$.

The test example for the hybrid scheme is shown in Fig. 4: a sudden start Couette flow. At $t=0$, the upper wall begins to move at the velocity $U_{w}=\sigma / \tau$ in $x$ direction when the bottom wall keeps fixed. The other two directions have the periodic boundary conditions. The distance between the two walls is $H=52.1 \sigma$ as in [14]. We separate the flow region with the unit area $\triangle x \times \triangle z=5.21 \sigma \times 5.21 \sigma$ in $x-z$ plane. The fluid is described as continuum hydrodynamics in the two-dimensional region between $z=z_{1}$ and $z=H$. This region occupies seven cells' length in $z$ direction. The fluid is described as particles between $z=0$ and $z=z_{2}$. This region occupies six cells' length in $z$ direction, and extends in $y$ direction with the cell length $\triangle$ 


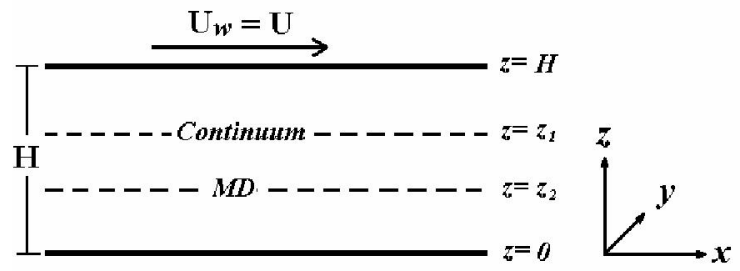

Figure 4. A schematic diagram of the Couette flow. $H$ denotes the distance between the two planar walls. $z=0$ denotes the bottom solid wall, $z=z_{1}$ the surface $P \rightarrow C, z=z_{2}$ the surface $C \rightarrow P, z=H$ the upper solid wall which starts to move at the constant velocity $U_{w}$ in the $x$ direction when $t=0$.

$y=4.81 \sigma$. The overlap region occupies three cells in $z$ direction and the constrained layer is located in the cells that are nearest to the interface $C \rightarrow P$. The bottom wall is simulated as two (111) planes of a face-centered cubic (fcc) lattice formed by solid particles. The positions of solid particles are fixed in the whole computational process. The interactions between fluid and solid particles are obtained by a shifted LJ potential

$$
V^{L J}(r)=4 \varepsilon^{w f}\left[\left(\frac{\sigma^{w f}}{r}\right)^{12}-\left(\frac{\sigma^{w f}}{r}\right)^{6}-\left(\frac{\sigma^{w f}}{r_{c}}\right)^{12}+\left(\frac{\sigma^{w f}}{r_{c}}\right)^{6}\right]
$$

with the density of the solid $\rho^{w}$. In the Couette flow, it has been proven that this shifted Lennard-Jones potential can yield different boundary conditions at the walls according to different groups of parameters [1]. The time step of the computation is $0.005 \tau$. We begin the simulation in the MD region. First, the program runs for $200 \tau$ to get a reasonable distribution of the positions and velocities for the fluid particles. Then the hybrid simulations were run for $2000 \tau$ to achieve a steady flow. To decrease the statistic fluctuations, we have averaged ten independent implements for each result.

Fig. 5 shows the velocity profiles at different time obtained by the hybrid method. The parameters of the wall particles in the hybrid method are $\varepsilon^{w f}=0.6 \varepsilon, \quad \sigma^{w f}=\sigma$ and $\rho^{w}=\rho$. Thompson [1] has shown that this group of parameters can achieve no-slip boundary conditions at the wall in the Couette flow. Thus, we can compare the results from the hybrid method with the analytical solutions from the NS equations with noslip boundary conditions. The velocity profiles are obtained by averaging the velocities in some time intervals. It can be seen that the results obtained from the hybrid method follow the ones obtained from the analytical solutions. And especially in the overlap region, the results from the two methods, MD simulations and NS equations' numerical solutions, are smoothly connected.

We also get the steady velocity profiles under different situations at the bottom wall. The upper wall is still in no-slip condition. The parameters presented in Fig. 6 are $\varepsilon^{w f}$, $\sigma^{\text {wf }}$ and $\rho^{w}$ in turn. The results from the hybrid method are well consistent with the full MD results. It indicates that the hybrid

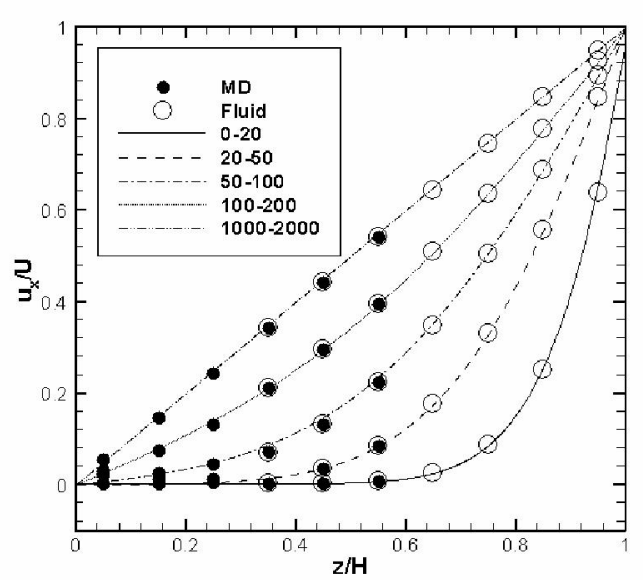

Figure 5. Velocity profiles at different time from the hybrid method and analytical solutions of the NS equations. Lines denote the analytical solution from the NS equations; Empty squares denote the result in the continuum region and solid circles denote the one in the particle region. The unit of time is $\tau$

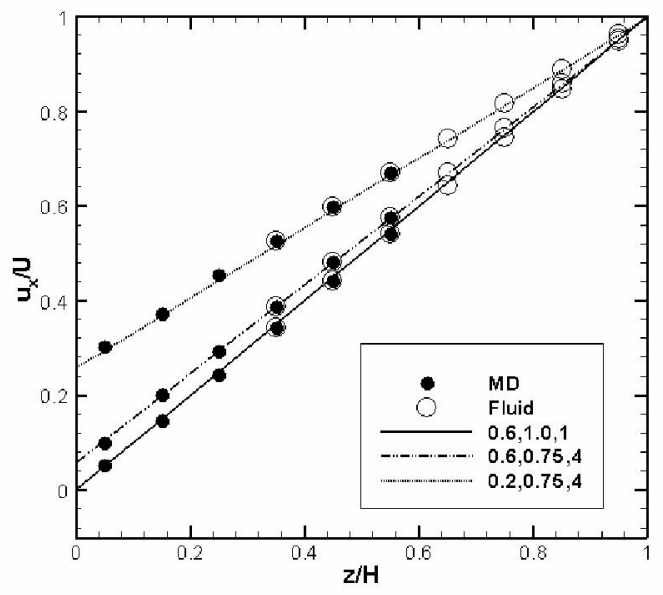

Figure 6. Steady velocity profiles of the Couette flows under no-slip and slip boundary conditions. The inlet indicates symbols of the results obtained from the hybrid method. Lines show the results obtained by full MD computations.

model is able to transfer the information from the wall to the fluid.

\section{CONCLUSIONS}

We have developed a dynamic Langevin model for the hybrid method of the MD and the NS equations, where the constrained particle dynamics is governed by the Langevin equation and the coupling parameter is dynamically adjusted by the constraint of velocity consistence. The coupling scheme has no free parameters, in the sense that the coupling parameters are self-adjusted in the computational process to fit 
the required dynamics. It is able to ensure the continuity of momentum. The hybrid method is used to simulate the suddenstart Couette flows with either slip or no-slip boundary conditions. The results obtained are in good agreement with the analytical solutions under the no-slip boundary conditions and the full MD simulations under the slip boundary conditions respectively. The dynamic Langiven model makes it practical to apply the hybrid method to various micro fluidic problems, and provides a novel methodology to determine the free parameters in the hybrid methods.

\section{REFERENCES}

[1] P. A. Thompson, and S. M. Troian, "A general boundary condition for liquid flow at solid surface," Nature, vol. 389, pp. 360-362, 1997.

[2] R. Pit, H. Hervet, and L. Leger, "Direct experimental evidence of slip in hexadecane: solid interfaces," Phys. Rev. Lett., vol. 85, pp. 980-983, 2000 .

[3] P. Koumoutsakos, "Multiscale flow simulations using particles," Annu. Rev. Fluid Mech., vol. 37, pp. 457-487, 2005.

[4] N. G. Hadjiconstantinou, "Hybrid atomistic-continuum formulations and the moving contact-line problem," J. Comp. Phys., vol. 154, pp. 245-265, 1995.

[5] N. G. Hadjiconstantinou, and A. T. Patera, "Heterogeneous atomisticcontinuum referesentations for dense fluid systems," Int. J. Mod. Phys., vol. 8, pp. 967-976, 1997.

[6] W. Q. Ren, and W. N. E, "Heterogeneous multiscale method for the modeling of complex fluids and micro-fluidics," J. comp. Phys., vol. 204 pp. 1-26, 2005.
[7] S. T. O'Connell, and P. A. Thompson, "Molecular dynamics-continuum hybrid computations: a tool for studying complex fluid flows," Phys. Rev. E, vol. 52, pp. 5792-5795, 1995.

[8] E. G. Flekkoy, G. Wagner, and J. Feder, "Hybrid model for combined particle and continuum dynamics," Europhys. Lett, vol. 52, pp. 271-276, 2000 .

[9] G. Wagner, E. G. Flekkoy, J. Feder, and T. Jossang, "Coupling molecular dynamics and continuum dynamics," Comp. Phys. Com.,vol.147, pp.670-673, 2002.

[10] G. Wagner, and E. G. Flekkoy, "Hybrid computations with flux exchange,” Phil. Trans. R. Soc. Lond. A, vol. 362, pp. 1655-1665, 2004.

[11] E. G. Flekkoy, J. Feder, and G. Wagner, "Coupling particles and fields in a diffusive hybrid model," Phys. Rev. E, vol. 64, pp. 066302 1-7, 2001.

[12] R. Delgado-Buscalioni, and P. V. Coveney, "Continuum-particle hybrid coupling for mass,momentum, and energy transfers in unsteady fluid flow," Phys. Rev. E, vol. 67, pp. 046704 1-13, 2003.

[13] R. Delgado-Buscalioni, E. G. Flekkoy, and P. V. Coveney, "Fluctuations and continuity in particle-continuum hybrid simulations of unsteady flows based on flux-exchange," Europhys. Lett., vol. 69, pp. 959-965, 2005 .

[14] X. B. Nie, S. Y. Chen, W. N. E and M. O. Robbins, "A continuum and molecular dynamics hybrid method for micro- and nano- fluid flow," $\mathbf{J}$. Fluid Mech., vol. 500, pp. 55-64, 2004.

[15] X. B. Nie, S. Y. Chen, and M. O. Robbins, "Hybrid continuum-atomistic simulation of singular corner flow," Phys. Fluids, vol.16, pp.3579-3591, 2004 